\title{
Molecular Evidence for Early Activity-Dependent Development of Hamster Motor Neurons
}

\author{
Robert G. Kalb and Susan Hockfield \\ Section of Neuroanatomy, Yale University School of Medicine, New Haven, Connecticut 06510
}

\begin{abstract}
Monoclonal antibody Cat-301 recognizes a surface antigen on subsets of neurons in many areas of the mammalian CNS, including Y-cells in the cat dorsal lateral geniculate nucleus (LGN) and spinal motor neurons in several species. We have shown previously that the Cat-301 antigen is expressed relatively late in postnatal development, with a time course that matches that of late developmental features of both LGN and spinal cord neurons. The developmental time course of the expression of the Cat-301 antigen in the LGN correlates with the period during which visual deprivation can alter LGN Y-cells. Neonatal visual deprivation (by monocular lid suture or dark rearing) suppresses Cat-301 expression on $\mathrm{Y}$-cells, while deprivation in adult animals has no effect on antigen expression. These results suggested that the onset of Cat301 immunoreactivity provides a positive molecular marker for the end of a critical period in cat visual system development.
\end{abstract}

To determine if Cat-301 might be a general marker for experience-dependent development in other areas of the CNS we have examined antigen expression on hamster spinal cord motor neurons. Here, we report that Cat-301 immunoreactivity develops on hamster motor neurons between postnatal days 7 and 14. Altering neuromuscular activity by sciatic nerve crush or thoracic hemicordotomy Inhibits Cat301 expression on motor neurons if performed before the onset of Cat-301 immunoreactivity. Other motor neuron antigens are unaffected by this procedure. In adult animals, nerve crush or cordotomy has no effect on Cat-301 immunoreactivity, demonstrating that Cat-301 expression is not simply dependent on ongoing neural activity. These observations suggest that motor neurons, like LGN neurons, require some pattern of neuronal activity during a critical period in development and, further, that the phenotypic changes in neurons consequent to early activity are reflected by the expression of specific molecules. The identification and characterization of such molecules may yield a description of the molecular mechanisms of experience-dependent development.

The precise connections among neurons in the mammalian CNS develop over an extended period of time. Many different mech-

\footnotetext{
Received June 16, 1987; revised Sept. 21, 1987; accepted Nov. 6, 1987.

This work was supported by grants to S.H. from NIH (EY-06511) and NSF (BNS-8544681), and to R. G. K. from NIH NS01247-01. S.H. is a Klingenstein fellow in the Neurosciences. We thank Marianne Bernstein for photographic assistance.

Correspondence should be addressed to Dr. Robert Kalb, Section of Neuroanatomy, Yale University School of Medicine, 333 Cedar Street, New Haven, CT 06510 .

Copyright (C) 1988 Society for Neuroscience $0270-6474 / 88 / 072350-11 \$ 02.00 / 0$
}

anisms together generate the final structure of the CNS. Some depend on intrinsic cellular properties (Banker and Cowan, 1979) or cell-cell interactions (Spemann, 1962). Others require interaction between the organism and its external environment (for review, see Wiesel, 1982). The landmark work of Hubel and Wiesel demonstrated that the morphology and physiology of neurons in the visual system can be profoundly influenced by an animal's visual experience early in life (Wiesel and Hubel, 1963; Hubel and Wiesel, 1985). Altering visual experience neonatally by monocular lid suture has permanent effects on normal cortical structure and function, while monocular suture in adult animals produces no permanent changes (Hubel and Wiesel, 1970). The period during which this experience-dependent development is sensitive to deprivation has been termed the critical period. The end of a critical period is reached when alterations of an organism's environment no longer generate permanent changes in neuronal anatomy or physiology. Much has been learned about these phenomena through single-cell recordings and histological techniques, but the molecular basis of experience-dependent development is just beginning to be explored.

Our approach to the study of experience-dependent development has been to use antibodies that discriminate between neurons in normal and experience-deprived animals. Work in this laboratory has identified a monoclonal antibody, Cat-301, that recognizes a surface molecule on subsets of neurons in the mammalian CNS (McKay and Hockfield, 1982; Hockfield and McKay, 1983; Hockfield et al., 1983). In the cat dorsal lateral geniculate nucleus (LGN), Cat-301 recognizes a distinct population of neurons, the Y-cells (Hockfield et al., 1983; Hendry et al., 1984; MacAvoy et al., 1985; Sur et al., 1988). During normal LGN development the expression of the antigen recognized by Cat-301 occurs postnatally as $Y$-cells acquire their mature morphological and electrophysiological properties (Hockfield et al., 1985; MacAvoy et al., 1985; Sur et al., 1988). The expression of the Cat-301 antigen on Y-cells is dependent on normal visual experience during the critical period. Animals monocularly deprived or dark reared from birth-procedures that reduce the number of physiologically identifiable $Y$-cells without affecting total cell number (Sherman et al., 1972; Friedlander et al., 1982)show a marked reduction in Cat-301 immunoreactivity in deprived layers of the LGN (Hockfield et al., 1985; Sur et al., 1988). Deprivation in adult animals does not reduce Cat-301 immunoreactivity, nor does it reduce the number of physiologically identifiable Y-cells, suggesting that Cat-301 expression is not simply dependent on continuous visually evoked neuronal activity. As early visual experience mediates the physiological maturation of Y-cells in the LGN, it also mediates a molecular event, the expression of the Cat-301 antigen. The onset of Cat301 expression correlates temporally with the end of the period 

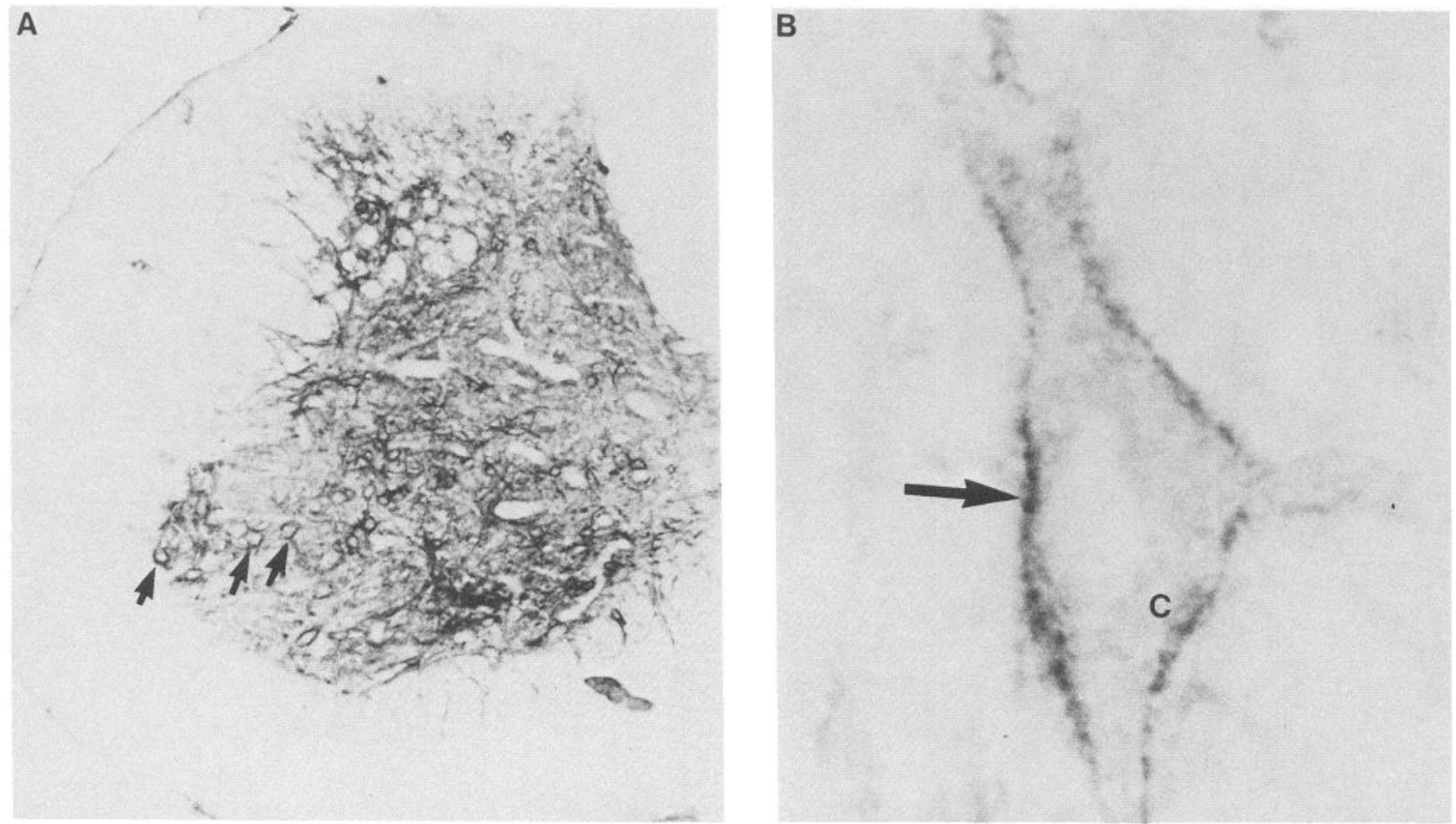

Figure 1. Cat-301 recognizes a surface antigen in hamster spinal cord. $A$, Low-magnification photomicrograph $(\times 140)$ of a $50-\mu \mathrm{m}-$ thick section of hamster spinal cord stained with Cat-301 (visualized using an HRP-conjugated second antibody). Several Cat-301-positive motor neurons are visible (arrows) in the ventral horn. B, At higher magnification $(\times 1290)$, the cytoplasm of a Cat-301-positive neuron is free of stain $(c)$, while the surface is intensely stained (arrow).

of LGN susceptibility to monocular deprivation and thus provides a positive molecular marker for experience-dependent development during a critical period.

Our aim in the present study was 2-fold: (1) to determine if Cat-301 expression might provide a general marker for early activity-dependent development in areas of the CNS in addition to the cat LGN; and (2) to establish a system in which to study the regulation of Cat-301 expression that was more accessible to manipulation and that had a shorter developmental time course than the cat visual system. The rodent spinal cord demonstrates major features of postnatal development during the first few weeks of life. In the hamster spinal cord monoclonal antibody Cat-301 recognizes motor neurons. We show here that the expression of the Cat-301 antigen on motor neurons in the hamster is dependent on an intact neuromuscular unit (motor neuron, muscle, segmental and suprasegmental afferents) in early life. Disrupting the neuromuscular unit neonatally inhibits Cat-301 expression on motor neurons, but disruption in the adult does not. The behavior of Cat-301 during development of spinal cord motor neurons parallels its expression in the LGN (Hockfield et al., 1985; Sur et al., 1988) in that the activity of an intact neuromuscular or visual system in early life is necessary for normal Cat-301 expression. In addition, these results provide evidence for a critical period in the development of motor neurons.

\section{Materials and Methods}

Animal care. Golden Syrian hamsters ranging in age from postnatal day 5 (P5) to P90 (adults) were reared in $12 \mathrm{hr:} 12 \mathrm{hr}$ light-dark cycle with $a d$ lib access to food and water. Newborn animals were handled daily before experimentation to desensitize the mother to the odor of human hands.

Fast blue labeling. Adult hamsters were anesthetized with pentobarbital sodium (10-15 mg/100 gm, intraperitoneally), and sciatic motor neurons were retrogradely labeled by injecting $2-3 \mu \mathrm{l}$ of $2 \%$ Fast blue (in water) into the sciatic nerve and then crushing the injection site. These procedures introduced Fast blue into sciatic motor neurons and axotomized the motor neurons. Motor neurons of P7 animals were labeled in the same manner as adults but under hypothermic anesthesia. To label sciatic motor neurons without damaging the sciatic nerve, an $8 \%$ solution of Fast blue was injected intramuscularly $(25 \mu 1 /$ muscle group in adults and $7.5 \mu \mathrm{l} /$ muscle group in neonates). The intramuscular injections were restricted to the dorsal thigh musculature, so only a subpopulation of the sciatic motor neuron pool was labeled using this procedure.

Hemicordotomies. Thoracic hemicordotomies were performed by dissecting away the paraspinal muscles over the thoracic vertebral spines and removing one set of posterior vertebral elements at the level of the interscapular fat pad. The cord was hemisected, taking care not to nick the dorsal midline vessel. A small piece of fat was placed over the laminectomy site and the wound sutured closed. In addition to routine postoperative care, the animal was checked for urinary retention by applying exogenous lower abdominal pressure until no urine could be expressed (twice daily).

Preparation of tissue and immunohistochemical techniques. The generation and characterization of the monoclonal antibodies used here have been described previously (Cat-301: McKay and Hockfield, 1982; Rat-302 and Rat-303: Hockfield, 1987). Tissue for immunohistochemistry was fixed by transcardial perfusion (under anesthesia) with $0.1 \mathrm{M}$ sodium phosphate-buffered saline, $\mathrm{pH} 7.4$ (PBS), followed by $4 \%$ paraformaldehyde in PBS. The lumbar enlargement was dissected into $30 \%$ sucrose in PBS. Longitudinal sections of the lumbar enlargement were cut on a cryostat at $30 \mu \mathrm{m}$. For immunohistochemistry, sections were incubated, free floating, in supernatant (full strength) from hybridoma cell lines, with $2 \%$ (vol/vol) Triton X-100 overnight, washed twice in PBS, and then incubated $4 \mathrm{hr}$ in either fluorescein isothiocynate-con- 

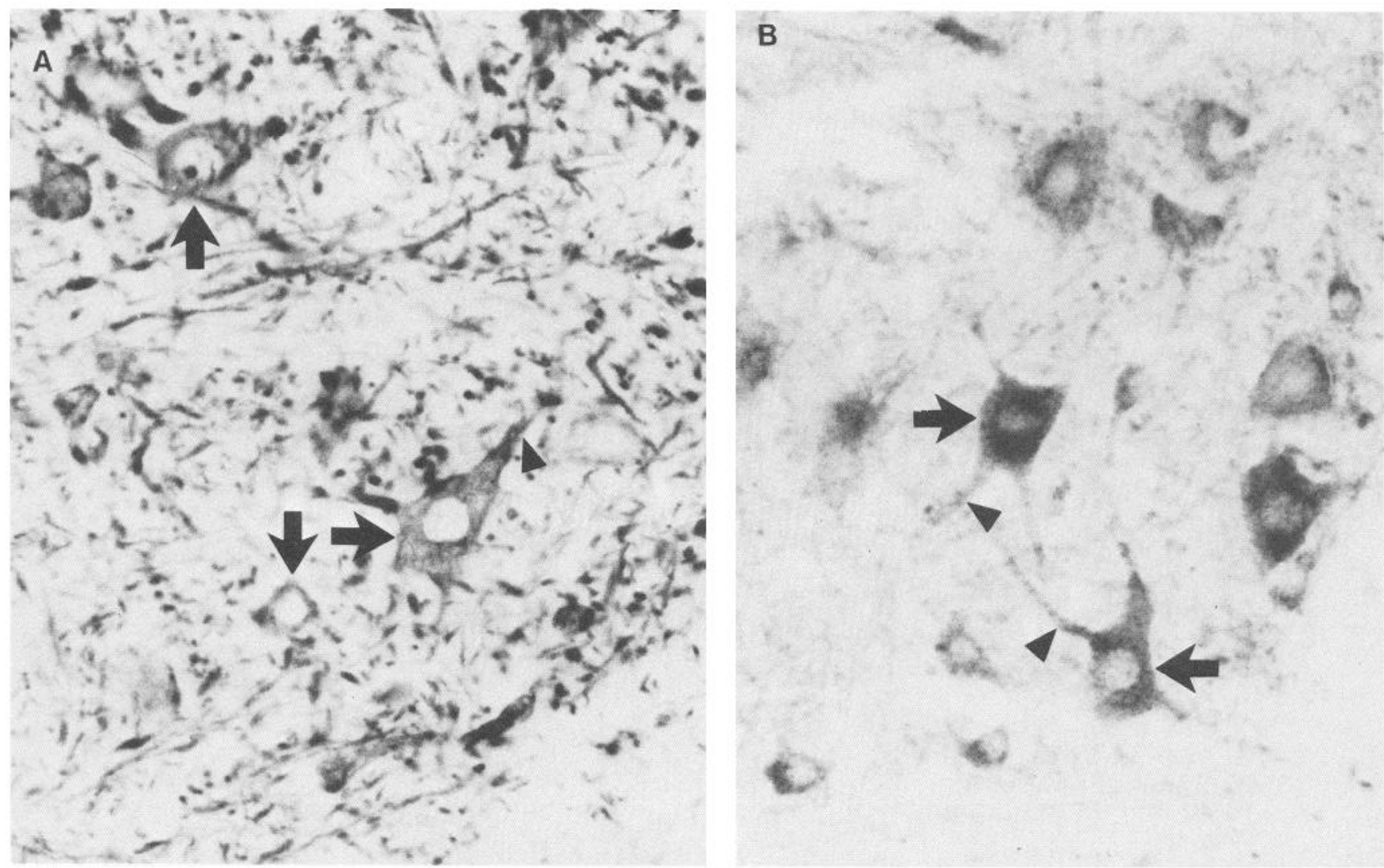

Figure 2. Antibodies Rat-302 and Rat-303 recognize intracellulaar antigens of motor neurons. $A$, Monoclonal antibody Rat-302 staining, visualized with HRP-conjugated second antibody, of adult motor neurons in the hamster spinal cord ventral horn. The cell bodies (arrows) and dendrites (arrowhead) are labeled. B, Monoclonal antibody Rat-303 staining of adult motor neurons (arrows) and their dendrites (arrowheads). While both antibodies recognize motor neurons, the subcellular localization of each is distinct. $\times 330$.

jugated (FITC-) or HRP-conjugated secondary antibody (goat anti-mouse IgG heavy and light chains, Cappel) diluted 1:100 in Dulbecco's modified Eagle's medium with $10 \%$ fetal calf serum, $0.2 \%$ sodium azide, and $2 \%$ Triton X-100. Following 2 PBS washes, HRP-labeled sections were treated with $3,3^{\prime}$-diaminobenzidine $(0.03 \%)$ with $\mathrm{H}_{2} \mathrm{O}_{2}(0.003 \%)$ to visualize the HRP. All sections were then mounted on gelatinized slides, dehydrated, cleared, and coverslipped.

Data collection. All experiments were performed at least in triplicate with animals from separate litters. To visualize Cat-301 and Rat-302, an FITC-conjugated secondary antibody was used, permitting simultaneous viewing of Fast blue with UV optics and FITC-labeled antigens with fluorescein optics. Rat-303 was visualized with an HRP-conjugated secondary antibody, as this gave a clearer signal for this antibody. A dense intracellular HRP-reaction product obscures the cytoplasmic Fast blue labeling of cells but not the nuclear labeling. Fast blue-positive cells were classified as positive or negative with regard to the specific antibody staining. Cell classification was equivocal in less than $5 \%$ of neurons sampled.

\section{Results}

\section{Cat-301 recognizes hamster spinal cord motor neurons}

Previous work from this laboratory has shown that monoclonal antibody Cat-301 recognizes subsets of neurons in many areas of the mammalian CNS, including the spinal cord of cats and primates but not of rats or mice (McKay and Hockfield, 1982; Hockfield et al., 1983). For the present study, we needed to define a neural system that (1) demonstrated Cat-301 immunoreactivity, (2) reached neuronal maturity relatively soon after birth, and (3) could be surgically manipulated neonatally. The hamster spinal cord provided such a system.

Monoclonal antibody Cat-301 recognized subsets of neurons in both the ventral and dorsal horns of the hamster spinal cord grey matter (Fig. 1A). Cat-301 binding sites (visualized with either an HRP- or FITC-conjugated secondary antibody) were distributed in an irregular, lacy pattern, outline the perimeter of cell bodies and proximal dendrites (Fig. $1 B$ ). This pattern of surface-associated staining matches that described previously in the cat spinal cord (McKay and Hockfield, 1982; Hockfield et al., 1983). At the electron microscopic level this staining pattern represents antibody binding sites present over much of the cell surface, in close association with presynaptic boutons but excluded from the synaptic cleft (Hockfield and McKay, 1983). Biochemical and ultrastructural data suggest that Cat301 recognizes a component of the extracellular matrix (Hockfield and McKay, 1983; Zaremba and Hockfield, 1987).

Two other monoclonal antibodies, Rat-302 and Rat-303 (Hockfield, 1987), also recognize hamster neurons and served as controls in our experiments. Monoclonal antibody Rat-302 recognized an intracellular antigen of ventral horn neurons and has a filamentous pattern throughout the cytoplasm (Fig. 2A). Monoclonal antibody Rat-303 also recognized an intracellular antigen in ventral horn neurons but gave a punctate staining pattern (Fig. 2B).

\section{All hamster sciatic motor neurons are Cat-301 positive}

Cat-301 bound to several different neuronal types in the hamster spinal cord (Fig. 1A). To carry out our experiments on the expression of the Cat-301 antigen, we needed to choose one group of Cat-301-positive neurons that could be reproducibly 

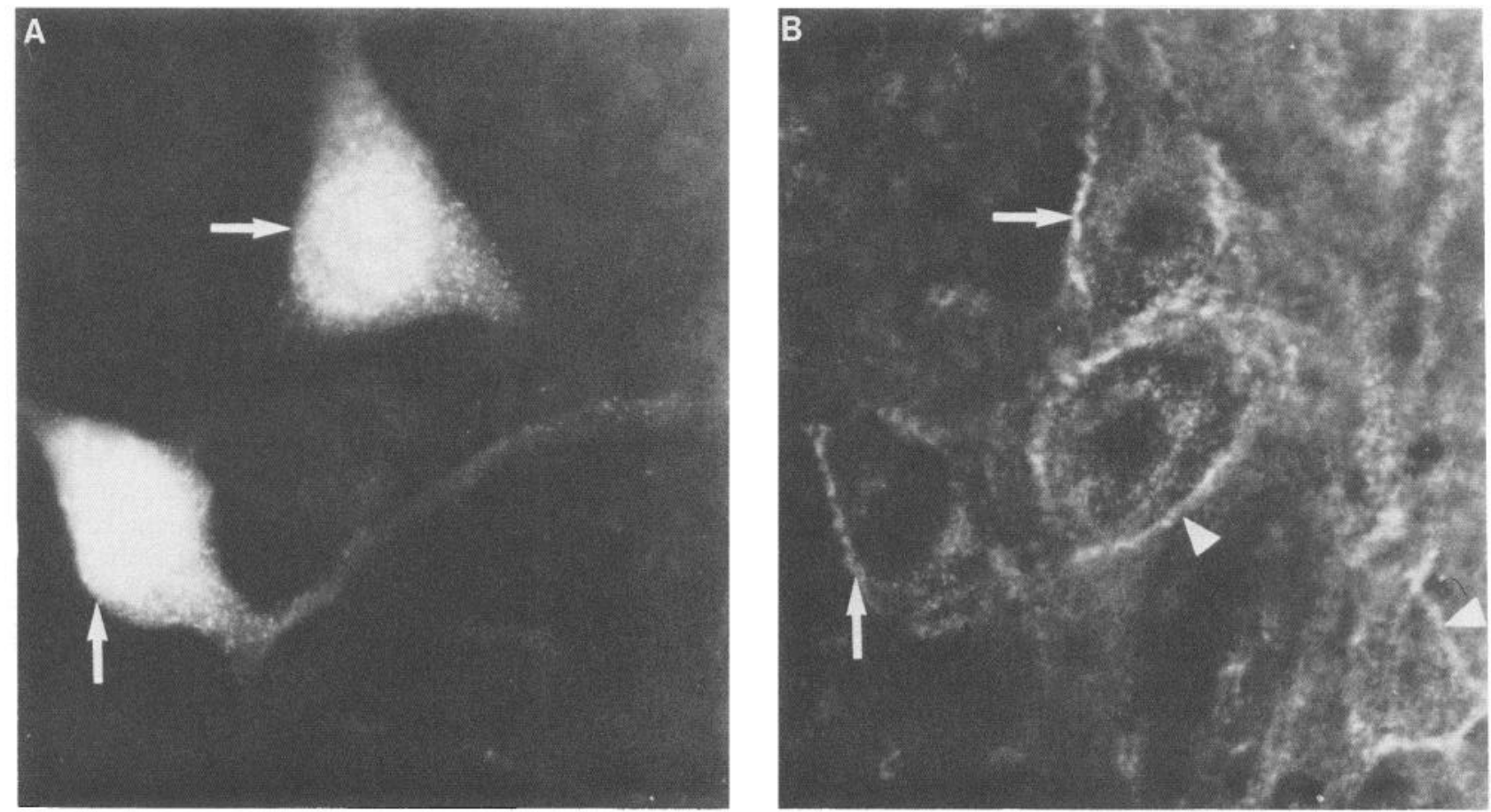

Figure 3. Following Fast blue injections into adult hamster sciatic nerve, retrogradely labeled motor neurons are Cat-301 positive. $A$, Fast bluecontaining cells (arrows) are visualized under UV illumination. The intracellular label has both a diffuse and a fine particulate quality, and nuclear stain brightly. $B$, Same field viewed under FITC optics shows Cat-301 staining. In this experiment, Cat-301 was visualized with an FITC-conjugated second antibody. The 2 Fast blue-labeled cells shown in $A$ are also Cat-301 positive (arrows). In addition, 2 neurons are shown that are Cat-301 positive (arrowheads) but Fast blue negative. $\times 655$.

identified and experimentally manipulated. The motor neurons that send axons in the sciatic nerve can be reliably labeled using the retrograde tracer Fast blue and so were selected for study. Injection of Fast blue into the sciatic nerve followed immediately by nerve crush produced an intracellular Fast blue signal in motor neurons with axons in the sciatic nerve (Fig. 3A). The Fast blue signal achieved its maximal intensity in $2 \mathrm{~d}$ and did not fade during survival periods of at least 2 months. In order to determine if either the labeling procedure or the intracellular presence of Fast blue had long-term toxic effects on motor neurons, we counted the number of Fast blue-positive cells present 2 and 4 weeks after labeling in the adult. The number of Fast blue-positive cells $(2440, \mathrm{SD}=312, n=6)$ was constant at all survival times.

Table 1. Number of sciatic motor neurons expressing Cat-301 following neonatal and adult lesions

\begin{tabular}{lll} 
& \multicolumn{2}{l}{ Cat-301-positive cells (\%) } \\
\cline { 2 - 3 } Group & P90 adults & $\begin{array}{l}\text { P21 } \\
\text { neonates }\end{array}$ \\
\hline Normal & 97 & 97 \\
Thoracic hemicordotomy & 99 & 49 \\
Sciatic nerve lesion & 98 & 4
\end{tabular}

The numbers reflect the proportion of the sciatic motor neuron pool that demonstrates Cat-301 immunoreactivity. In adult animals, virtually all sciatic motor neurons express Cat-301 after thoracic hemicordotomy or sciatic nerve lesion, while in neonates, there is a significant reduction in the number of Cat301-positive motor neurons after the same lesions. A minimum of 300 cells were counted in each of at least 2 animals to derive percentages.
To determine whether all sciatic motor neurons express the Cat-301 antigen as well as the antigens recognized by antibodies Rat-302 and Rat-303, we combined immunohistochemistry with Fast blue labeling. In adult animals, $2 \mathrm{~d}$ after intranerve Fast blue injection and nerve crush, all labeled sciatic motor neurons exhibited Cat-301 immunoreactivity (Table 1, Fig. 3B). There were, of course, many Cat-301-positive spinal cord neurons not labeled by these injections. Based on their shape and location, these Fast blue-negative, Cat-301-positive neurons appeared to be interneurons and motor neurons that did not have axons in the sciatic nerve. Since there is no way to reproducibly identify and therefore quantitate these cells, we were unable to evaluate the effect of our experimental manipulations on Cat-301 expression on these Fast blue-negative cells. The same double-labeling techniques showed that all Fast blue-positive sciatic motor neurons also expressed the intracellular antigens recognized by antibodies Rat-302 and Rat-303 in adult animals.

\section{Sciatic motor neurons express adult levels of Cat-301 by P14}

The Cat-301 antigen is expressed postnatally in the cat spinal cord (Hockfield et al., 1983) and LGN (Hockfield et al., 1985; Sur et al., 1988). As a first step in testing whether the expression of Cat-301 on motor neurons might be regulated by early activity, we needed to determine the developmental time course of Cat-301 expression on sciatic motor neurons. Motor neurons were retrogradely labeled by injecting selected muscles innervated by the sciatic nerve with Fast blue. Among this backlabeled subpopulation of sciatic motor neurons, none were Cat301 positive at $\mathrm{P} 7$, and virtually all were Cat-301 positive by P14. Companion experiments have shown that the antigens 
recognized by Rat-302 and Rat-303 were also expressed within the first 2 postnatal weeks.

\section{Sciatic nerve lesion or thoracic cord lesion has no effect on Cat-301 expression in adults}

In order to determine if Cat-301 expression on motor neurons is dependent on some component of neural activity, adult and neonatal sciatic motor neurons were deprived of normal neuromuscular activity. In our initial studies, we chose a relatively severe form of deprivation, surgically cutting or crushing the sciatic nerve. These lesions destroy both the axons of motor neurons as they course from the spinal cord to the limb and the afferents that travel in the sciatic nerve. The procedure we used to label sciatic motor neurons with Fast blue (by intranerve injection and nerve crush) also lesioned the sciatic nerve.

Two weeks after sciatic nerve lesion (and Fast blue labeling) in adults, all dye-positive motor neurons were Cat-301 positive (Table 1). The motor neuron antigens recognized by antibodies Rat-302 and Rat-303 were also present in all scialic motor neurons 2 weeks after a sciatic nerve lesion. The continued presence of the 3 antigens 2 weeks after sciatic nerve lesions showed that continued antigen expression in adults is not dependent on effective neuromuscular activity.

In an effort to disrupt normal neuromuscular activity without directly damaging the motor neuron, we next unilaterally interrupted suprasegmental influences on the sciatic motor neurons by hemisection of the thoracic spinal cord. To verify the thoracic cord lesion, spinal cord sections through the region of the lesion were examined with a monoclonal antibody, Cat-101, that recognizes intact axons (Hockfield and McKay, 1985) and with a Nissl stain. Thoracic hemicordotomy has pronounced effects on neuromuscular activity, as cordotomized animals drag the paretic limb or walk on the dorsum of the affected paw. The effect of thoracic cord lesions on Cat-301 expression by sciatic motor neurons was assayed by Fast blue labeling of motor neurons ipsilateral to the cordotomy $12 \mathrm{~d}$ after a thoracic cord lesion and $2 \mathrm{~d}$ prior to sacrificing the animal. Our previous experiments showed that the Fast blue labeling procedure alone had no effect on Cat-301 expression. Two weeks after spinal cord hemisection in the adult, all sciatic motor neurons continued to express Cat301 immunoreactivity (Fig. 4, $A, B$ ). Similarly, hemicordotomy had no effect on Rat-302 or Rat-303 immunoreactivity (Table 1; Fig. 4, $C-F$ ), demonstrating that loss of effective neuromuscular activity in adults has no influence on the antigens recognized by any of these antibodies. These results, together with those of the sciatic nerve lesions, show that Cat-301 expression on adult motor neurons is not simply activity-dependent.

As we do not yet know the turnover rate of the Cat-301 antigen, it is possible that the persistent staining observed in adult animals following lesions might reflect either a short halflife with continucd synthesis at normal levels or a long half-life combined with antigen stability to lesion effects. Observations of Cat-301 expression in cats favor the former possibility, as monocular-deprivation in adults produces no appreciable diminution of staining even after 6 months. It is doubtful that antigen turnover would be slow enough to account for these findings. Similarly, our preliminary data suggest that Cat-301 labeling of adult hamster motor neurons is not appreciably diminished 1 month after lesioning.
Neonatal disruption of neuronal activity suppresses the development of Cat-301 immunoreactivity on sciatic motor neurons

To determine if effective neuromuscular activity was necessary for the development of Cat-301 expression, sciatic nerve crush was performed at P7, prior to Cat-301 expression. As in the experiments described above in adult animals, the sciatic nerve of P7 animals was injected with Fast blue and crushed; 2 weeks later, at P21, the lumbar cord was examined for Cat-301 immunoreactivity. As in the adult, we counted the number of Fast blue cells after the labeling procedure $(2,7,14,28$, and $56 \mathrm{~d})$ and found the number of labcled cells was less than in the adult, but the number remained constant over time (1101, $\mathrm{SD}=55$, $n=5$ ). The rostrocaudal and dorsoventral distributions of Fast blue-positive cells in longitudinal sections appeared the same at all survival times in the neonate as in the adult. The smaller number of Fast blue cells found in neonates may be related to incomplete labeling of cells due to the difficulty of injecting the P7 sciatic nerve with the dye. An alternative possibility is that axotomy induces cell death in a subpopulation of sciatic motor neurons (Schmalbruch, 1984). As the number of labeled cells remains constant from 2 to $28 \mathrm{~d}$ after axotomy, any cell death that may occur must represent an acute effect of the lesion.

In marked contrast to adults, following a sciatic nerve lesion at P5 sciatic motor neurons did not express the Cat-301 antigen 2 weeks later, at P21 (Table 1, Fig. 5). As sciatic nerve lesions were performed unilaterally, the opposite, unlesioned side served as a control. Greater than $97 \%$ of sciatic motor neurons on the unlesioned side were Cat-301 positive, while not more than $4 \%$ on the lesioned side were positive. Given the pronounced effects on Cat-301 expression within the population of sciatic motor neurons labeled in the neonate, cell death is unlikely to play a significant role in our observations.

In order to determine if this loss of Cat-301 immunoreactivity was a relatively specific result of the lesion or if it reflected a generalized decrease in protein synthesis, alternate sections were examined for Rat-302 and Rat-303 immunoreactivity. Following unilateral sciatic nerve lesions, all Fast blue-positive motor neurons were immunoreactive for the Rat-302 and Rat-303 antibodies. Deprivation of neuromuscular activity by axotomy early in development selectively prevented the expression of the Cat-301 antigen, leaving other antigens unaffected.

As described in the previous section, another way to block effective neuromuscular activity (without directly damaging the motor neuron) is to interrupt descending input to the ventral horn by lesioning the thoracic spinal cord. Thoracic hemicordotomies were performed on neonates prior to the expression of Cat-301 on sciatic motor neurons. The thoracic spinal cord was hemisected on $\mathrm{P} 7$, followed by retrograde labeling of sciatic motor neurons on P19, $2 \mathrm{~d}$ before sacrificing the animal. We previously demonstrated that lesioning the sciatic nerve at P19 alone (to permit retrograde labeling of sciatic motor neurons) had no effect on motor neuron survival or on Cat-301 immunoreactivity. Here again, in dramatic contrast to the adult, thoracic hemicordotomy at $\mathrm{P} 7$ resulted in a significant reduction in the number of Cat-301-positive sciatic motor neurons on the lesioned side of the cord (Table 1; Fig. 6, $A, B$ ). Because severely neurologically impaired neonates are rejected by their mothers and do not survive, all hemicordotomies were incomplete and 

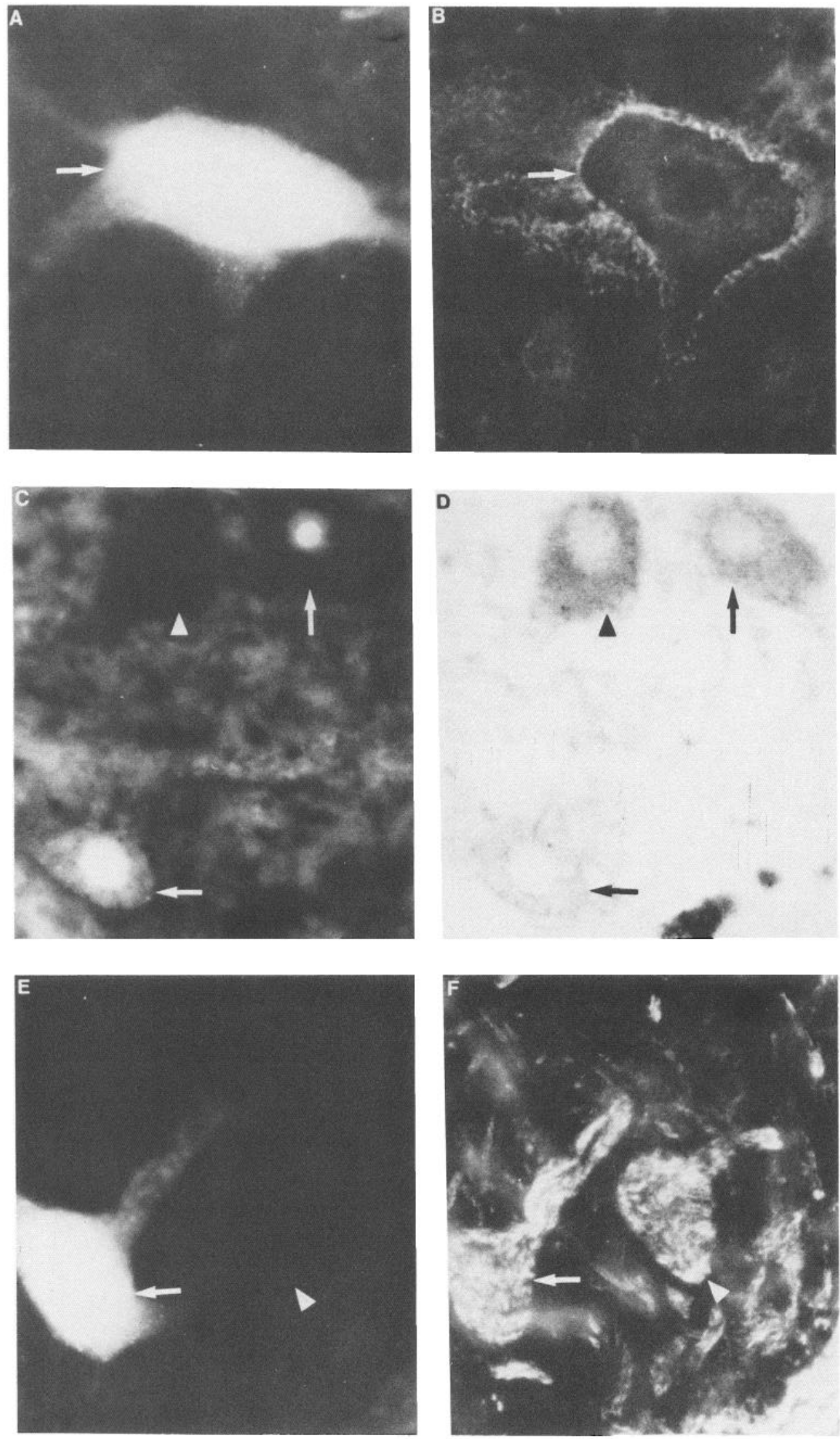

Figure 4. Two weeks after thoracic hemicordotomy, all adult sciatic motor neurons continue to express Cat-301, Rat-303, and Rat-302. Twelve days after thoracic cord lesion and 2 days prior to sacrificing the animal, the sciatic motor neurons were back-labeled with Fast blue from the sciatic nerve. $A$, Sciatic motor neurons show a homogeneous, particulate intracellular staining under UV illumination. $B$, The section shown in $A$ was stained for Cat-301 (using an FITC second antibody). The same field as in $A$ viewed under FITC optics shows that Cat-301 labels the Fast blue-positive sciatic motor neuron shown in $A$. The cell body (arrow) and proximal dendrites have typical surface-associated Cat-301 staining. At the FITC excitation wavelength a small amount of Fast blue stain is visible intracellularly as a faint blue hue. $C$, Two Fast bluepositive sciatic motor neurons viewed under UV optics (arrow) in a section that has been stained with Rat-303 and an HRP-conjugated second antibody. The cytoplasmic HRP quenches the Fast blue fluorescence, while the nuclear label is still clearly visible. $D$, Same field shown in $C$ viewed under bright field illumination for Rat-303 immunoreactivity visualized with HRP-conjugated second antibody. Two Fast blue-positive sciatic motor neurons are Rat-303 positive (arrows) and another Fast bluenegative, Rat-303-positive cell is also shown (arrowhead). E, Fast blue-positive sciatic motor neuron (arrow) in another section, counterstained for Rat302. $F$, Same field as in $E$ viewed under FITC optics. Rat-302 immunoreactivity visualized with FITC-conjugated second antibody has a filamentous staining at the location of the Fast bluepositive cell (arrow) shown in $E$, as well as in a Fast blue-negative neuron ( $a r$ rowhead). $\times 870$. 

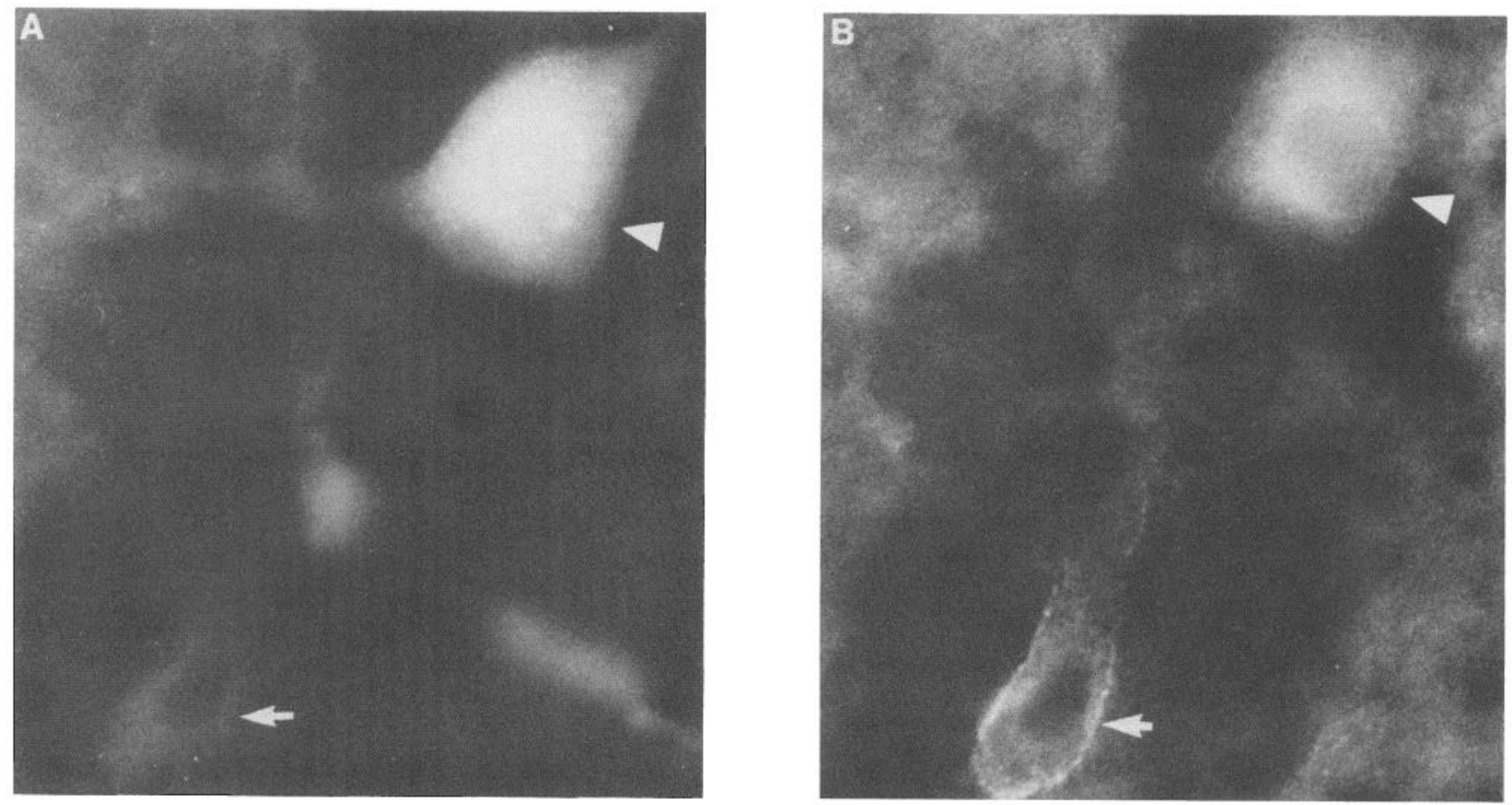

Figure 5. Two weeks after neonatal sciatic nerve lesion at P7, retrogradely labeled motor neurons do not express Cat-301 immunoreactivity. $A$, One motor neuron in this field shows retrograde labeling with Fast blue (arrowhead), 2 weeks after a sciatic nerve lesion. The UV illumination faintly excites the FITC labeling of a Cat-301-positive, Fast blue-negative cell in the lower portion of the panel (arrow). Under UV illumination this cell fluoresces a faint yellow-green. $B$, Same field shown in $A$ viewed under FITC optics. No surface-associated Cat-301 immunoreactivity (visualized with FITC-conjugated second antibody) is seen at the location of the Fast blue cell (arrowhead). As this photomicrograph has been overexposed to more clearly demonstrate the lack of Cat-301 immunoreactivity, the fine intracellular particulate Fast blue staining is seen breaking through under FITC illumination. A Fast blue-negative, Cat-301-positive cell is seen in the lower portion of the field (arrow). $\times 930$.

approximately $50 \%$ of sciatic motor neurons express Cat-301. On the control, unlesioned side of the cord, sciatic motor neurons expressed normal levels of Cat-301 immunoreactivity. As with the sciatic nerve lesions, there was no discernible effect of hemicordotomy on the motor neuron antigens recognized by Rat-302 or Rat-303 on lesioned or control sides (Fig. 6, $C-F$ ). In these experiments we could not determine if there was an effect of thoracic cord lesion on Cat-301 expression in Fast bluenegative cells because we have no way of reproducibly identifying them. These experiments demonstrated that impairing the use of a limb neonatally with an upper motor neuron lesion inhibited the expression of the Cat-301 antigen.

\section{Discussion}

Early neuromuscular activity regulates the expression of the Cat-301 antigen on sciatic motor neurons.

In this report we show that the expression of the antigen recognized by monoclonal antibody Cat-301 on sciatic motor neurons requires normal neuromuscular activity early in development. The Cat-301 antigen was expressed postnatally on motor neurons between days 7 and 14 of life. Disruption of motor neuron activity neonatally by sciatic nerve lesion or thoracic cord hemisection suppressed Cat-301 immunoreactivity, whereas the same procedures in adult animals had no effect on Cat-301 expression. These findings are similar to our results on the experience-dependent expression of Cat-301 in the visual system, where alteration of the activity of LGN neurons in early life inhibits Cat-301 expression (Hockfield et al., 1985; Sur et al., 1988).

Previous experiments have demonstrated experience-depen- dent development in the mammalian CNS during a circumscribed period of time, called the critical period (Hubel and Wiesel, 1970). During the critical period, perturbation of the normal pattern of neural activity has permanent, measurable effects on the anatomy and physiology of CNS neurons. For example, the normal segregation of thalamocortical afferents in layer IVB of the cat striate cortex is significantly altered by neonatal monocular lid suture during the first 3 postnatal months (Wiesel and Hubel, 1963; Hubel and Wiesel, 1970). Lid suture in adults does not affect the ocular dominance organization. Other areas of the visual system in addition to layer IVb of cortical area 17 also exhibit experience-dependent development, including the LGN and other layers of area 17 (Sherman et al., 1972; Wiesel, 1982).

Experience-dependent development may be mediated by neuronal electrical activity. In the visual system, action potential blockade by intraocular administration of the sodium channel blocker TTX prevents normal segregation of left and right eye inputs to the fish tectum (Meyer, 1983), cat LGN (Archer et al., 1982), and cat cortex (Stryker and Harris, 1986). Alterations in the pattern of neuronal electrical activity are likely to underlie the abnormal development consequent to monocular lid suture and intraocular TTX, as well as the abnormal development demonstrated in the present study consequent to surgical lesions of components of the spinal neuromuscular unit.

The molecular biology of experience-dependent development is just beginning to be explored and may be facilitated by the use of hybridoma technology. Monoclonal antibody Cat-301 recognizes a surface molecule on LGN Y-cells whose expression is experience-dependent. Deprivation experiments suggest that 

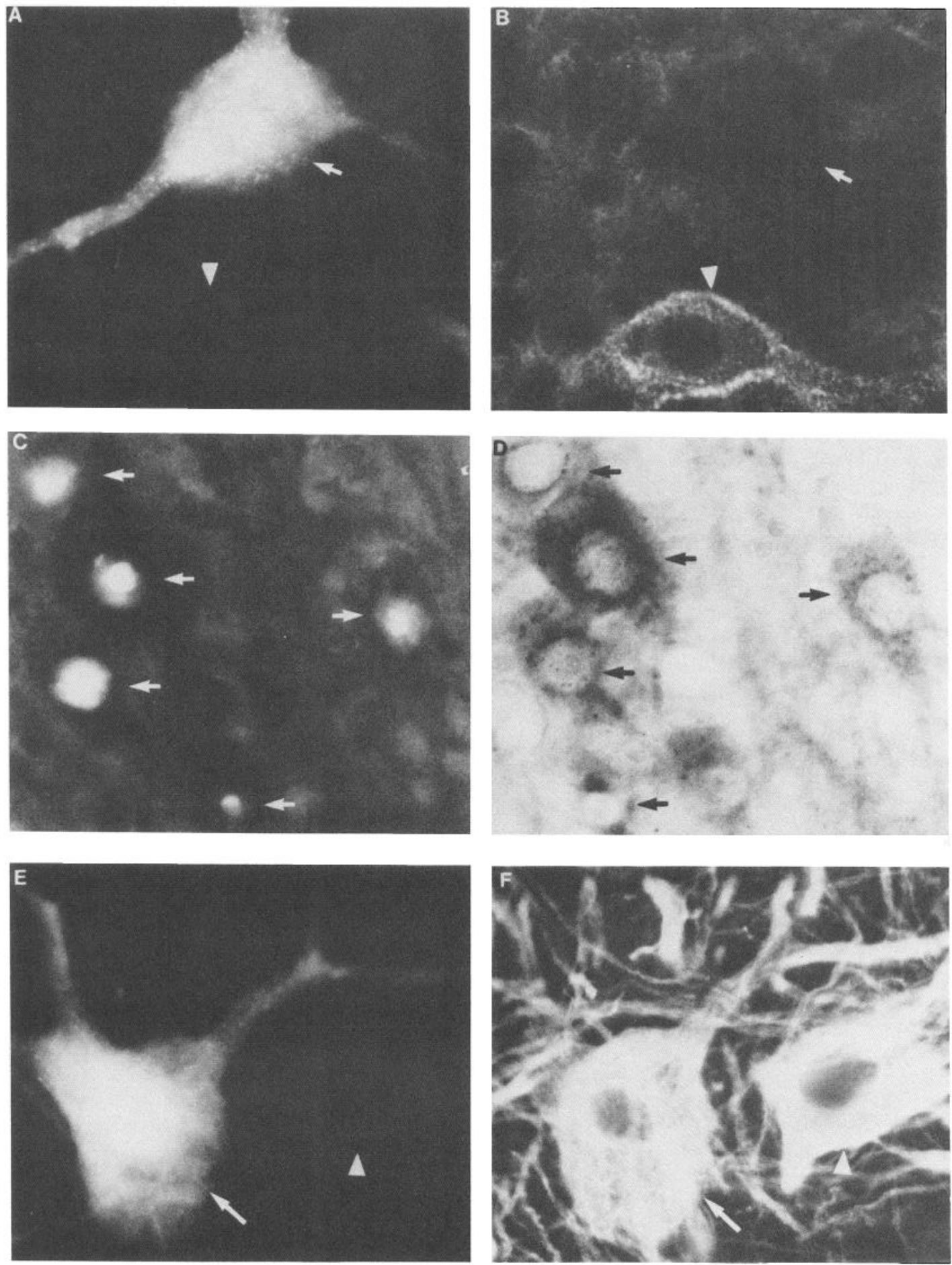

Figure 6. Two weeks after thoracic hemicordotomy at P7, sciatic motor neurons do not express Cat-301 but do express Rat-302 and Rat-303. Twelve days after thoracic cord lesion, and $2 \mathrm{~d}$ prior to sacrificing the animal, the sciatic motor neurons are visualized by injection of Fast blue into the sciatic nerve. $A$, Motor neuron seen here (arrow) has particulate staining on a homogeneous background under UV illumination. A small amount of Cat-301 FITC labeling bleeds through under UV illumination (arrowhead) as a yellow-green outline of a Fast blue negative cell (arrowhead). $B$, Same field viewed under FITC optics shows that Cat-301 (visualized with an FITC-conjugated 2nd antibody) labels a Fast blue negative cell (arrowhead). No Cat-301 staining is seen of the Fast blue cell shown in $A$ (arrow). $C$, Five Fast blue positive sciatic motor neurons are seen in this field (arrows). D, Same field shown in $C$ viewed under bright field illumination. Rat-303 visualized with HRP-conjugated 2 nd antibody labels all 5 Fast blue labeled motor neurons. $E$, A Fast blue positive sciatic motor neuron viewed under UV illumination (arrow). $F$, Same field shown in $E$ viewed under FITC optics. Rat-302 immunoreactivity visualized with FITC-conjugated 2nd antibody has a filamentous staining over the location of the Fast blue positive cell shown in $E$ (arrow), and of a Fast blue negative cell (arrowhead). $\times 800$. 
the onset of the expression of Cat-301 on Y-cells correlates with the end of the previously defined critical period for these cells and may provide a positive molecular marker for experiencedependent development (Hockfield et al., 1985; Sur et al., 1988). Two other studies have shown that early experience-deprivation affects the molecular characteristics of neurons in visual pathways. Visual deprivation of cats from birth produces a reduction in the complexity of mRNA in the visual cortex (Grouse et al., 1979) and alters the state of phosphorylation of microtubuleassociated proteins in striate cortex but not LGN (Aoki and Siekevitz, 1985). Together these and the present study have begun to describe the molecular changes that may subserve the previously documented anatomical and physiological effects of early neural activity on neuronal development.

Two independent deprivation paradigms reduce Cat-301 immunoreactivity in neonates but not in adults

An important feature of the present study is our demonstration that 2 independent methods of depriving animals of normal early neuromuscular activity both reduce Cat-301 immunoreactivity. The first experiments were designed to determine if we could demonstrate an effect on the Cat-301 antigen by any sort of deprivation. Therefore, to begin we chose a relatively severe deprivation procedure, cutting the sciatic nerve to lesion both motor neuron axons and sciatic sensory afferents. The sciatic nerve lesions in neonates, but not in adults, suppressed Cat-301 immunoreactivity. One might argue that axotomy could be predicted to have an effect on the motor neuron's molecular propertics, as it has demonstrable effects on neonatal and adult neuronal morphology (Ramon y Cajal, 1928; Blinzinger and Kreutzberg, 1968). However, the different responses of Cat-301 expression to sciatic nerve lesion in neonate and adult indicate that the regulation of this molecule is independent of axotomyinduced chromatolysis.

One concern in our experiments was that fewer cells are retrogradely labeled in the neonate than the adult. We are unable to distinguish technical difficulties in labeling from axotomy-induced cell death. Three lines of evidence suggest that the neonatally labeled motor neurons are both healthy and representative of the adult sciatic motor neuron pool: (1) The distribution of labeled cells in the spinal cord is the same in neonates and adults; (2) there is no decline in the number of neonatally labeled cells up to 4 weeks after sciatic nerve lesion; and (3) the expression of the control antigens, Rat-302 and Rat-303, are unaffected, suggesting no gencral declinc in protcin synthesis after sciatic nerve lesion.

Our next experiments were designed to test whether the suppression of Cat-301 immunoreactivity on motor neurons following neonatal sciatic nerve lesions might be tied to a general loss of effective neuromuscular activity, rather than to some effect of direct neuronal injury. We then subjected animals to thoracic hemicordotomy, a form of deprivation that did not involve direct injury to the neurons under study but did dramatically alter the pattern of neuromuscular activity of the sciatic motor unit. Neonatal hemicordotomy, like neonatal sciatic nerve lesion, also reduced Cat-301 immunoreactivity without having other demonstrable effects on motor neuron morphology or molecular composition.

We do not yet know if these 2 deprivation procedures act through separate or common pathways to achieve the effects we report here. Certainly we can rule out shared direct effects of these lesions, as they involve completely independent groups of axons. It is possible that both lesions secondarily involve a shared class of spinal cord interneuron that might receive input from the descending axons (lesioned by cordotomy) and the segmental afferents (lesioned by cutting the sciatic nerve) and then, in turn, synapse on the motor neurons. Our current ultrastructural studies will resolve whether a common class of synapses on motor neurons is affected by both lesions.

The lack of change of Cat-301 expression following adult deprivation of motor neurons or $\mathrm{Y}$-cells stands in contrast to other studies that have reported changes in the levels of various enzymes following adult deprivation (Graybiel and Ragsdale, 1982; Wong-Riley and Carroll, 1984; Hendry and Kennedy, 1986). These enzymes are thought to change relative to the level of neuronal activity. Cat-301 does not appear to behave in this manner, suggesting that its expression is not simply tied to neuronal activity, but to developmental processes of neurons in early postnatal life.

\section{Motor neurons may have a critical period in development}

The time course of Cat-301 expression on motor neurons and its time-limited susceptibility to lesions of the neuromuscular unit demonstrate that a critical pcriod cxists for motor neuron development. In the present experiments, the end of the critical period for motor neurons occured at least by P90; our ongoing experiments will allow us to determine more precisely the outer limit of its time course. To our knowledge this is the first demonstration that early postnatal activity is essential for the molecular development of motor neurons.

In this report we have limited our observations to short survival periods following the lesions. Other studies have shown that after longer survival periods motor neurons can successfully reinnervate muscles. Some of the features of neurons during reinnervation match those of neurons during development (McArdle, 1975). We do not yet know if during reinnervation the expression of the Cat-301 antigen will recapitulate its behavior during development.

The early activity-dependent expression of Cat-301 on motor neurons occurs at a time of active morphological, physiological and neurochemical changes within the spinal cord. Previous reports have shown that early in development the number of axons (Chung and Coggeshall, 1984) and synapses in several areas of the spinal cord exceeds that observed in the adult and declines during early postnatal life. Up to $50 \%$ of the initial synapses on motor neurons are lost in the first few weeks of life (Ronnevi and Conradi, 1974; Conradi and Ronnevi, 1975). In addition, during this period axonal boutons on motor neurons undergo qualitative changes in synapse type (Conradi, 1969; Conradi and Ronnevi, 1977). A similar reduction and modification of synapses is suggested by the excessive development of dendritic spines on rat motor neurons and subsequent reduction to adult levels within the first 3 weeks of life (Cummings and Sielzner, 1984).

Biochemical changes during postnatal spinal cord development have also been documented. In the rat, the synthesis of norepinephrine in the ventral horn rises to its peak at $20 \mathrm{~d}$ of life (most rapidly in the first $4 \mathrm{~d}$ ) and subsequently declines to adult levels, suggesting rapid postnatal proliferation and then modification of noradrenergic input to the cord (Commissiong, 1984). Postnatal development of choline acetyltransferase (ChAT) in motoneurons of the rat spinal cord is most marked during the first 14-21 d of life, with subgroups of cholinergic neurons attaining adults levels of ChAT at different times (Phelps 
et al., 1984). The effects of an animal's early experience on norepinephrine and ChAT have not been reported.

The importance of intact segmental spinal cord circuitry in early life on neuronal development has been demonstrated in both the dorsal and ventral horn. Unilateral dorsal rhizotomy in 3- to 5-d-old kittens results in a hypoplasia of the neurons that would normally receive the removed input. In addition, the hypoplastic neurons have highly abnormal dendritic arbors. The same procedure performed on older animals (more than 7 $\mathrm{d}$ of age) has no discernible effect on cell size or dendritic morphology (Smith, 1974). These results provide evidence that a critical period in development exists in the dorsal horn during which loss of neural activity carried by afferent fibers causes permanent structural changes in spinal cord neurons. In the ventral horn a series of critical periods has been proposed based on the demonstration that axotomized neurons can successfully reinnervate muscle only during circumscribed periods (Dennis and Harris, 1980; Sheard et al., 1984). Prenatal and early postnatal critical periods may correlate with the times of initial synaptogenesis and of synapse elimination and may depend on intact connections between motor neuron and muscle.

The behavior of the antigen recognized by Cat-301 on motor neurons also provides evidence for a critical period in motor neuron development. The developmental process reflected by the expression of Cat-301 immunoreactivity is distinct from previously described early developmental processes (Dennis and Harris, 1980; Sheard et al., 1984) in that it depends on intact segmental and suprasegmental connections. This dependence on complex circuitry suggests that a specific balance, or pattern, of motor neuron activity in early life is essential for maturation. In this way, motor neuron development may demonstrate experience-dependent developmental features similar to those observed in the visual system, where patterned activity is required for the maturation of LGN Y-cells, including the expression of Cat-301 immunoreactivity. Cat-301 thus provides a reagent with which to test the effects of pharmacological and surgical manipulations on early motor neuron development. In addition, corrclations betwecn particular synaptic cvents and Cat-301 expression may define a critical period at the single cell level. Ultimately these studies may shed light on the functional role played by the Cat-301 molecule. The timing of its expression, its subcellular localization (Hockfield and McKay, 1983), and its characterization as a proteoglycan (Zaremba and Hockfield, 1987) suggest it might participate in synapse stabilization.

The late onset of Cat-301 immunoreactivity and its regulation by neuromuscular (or visual) activity suggest that critical postnatal features of development are also reflected in changes in the molecular properties of neurons. The identification and characterization of such molecules holds the promise that the molecular biology of experience-dependent development during a critical period is accessible to study.

\section{References}

Aoki, C., and P. Siekevitz (1985) Ontogenetic changes in the cyclic adenosine 3',5'-monophosphate stimulatable phosphorylation of cat visual cortex proteins, particularly of microtubule-associated protein 2 (MAP 2): Effects of normal and dark rearing and of the exposure to light. J. Neurosci. 5: 2465-2483.

Archer, S. M., M. W. Dubin, and L. A. Stark (1982) Abnormal development of kitten retino-geniculate connectivity in the absence of action potentials. Science 217: 743-745.

Banker, G. A., and W. M. Cowan (1979) Further observations on hippocampal neurons in the dispersed cell culture. J. Comp. Neurol. 187: 469-494.

Blinzinger, K., and G. Kreutzberg (1968) Displacement of synaptic terminals from regenerating motoneurons by microglial cells. Z. Zellforsch. 85: 145-157.

Chung, K., and R. E. Coggeshall (1984) The postnatal development of the tract of Lissauer in the rat. J. Comp. Neurol. 229: 471-475.

Commissiong, J. W. (1984) Synthesis of catecholamines in the developing spinal cord of the rat. J. Neurochem. 42: 1574-1581.

Conradi, S. (1969) Observations on the ultrastructure of the axon hillock and initial axon segment of lumbosacral motoneurons in the cat. Acta Physiol. Scand. 332: 65-84.

Conradi, S., and L-O. Ronnevi (1975) Spontaneous elimination of synapses on cat spinal motoneurons after birth: Do half of the synapses on the cell bodies disappear? Brain Res. 92: 505-510.

Conradi, S., and L-O. Ronnevi (1977) Ultrastructure and synaptology of the initial axon segment of cat spinal motoneurons during early postnatal development. J. Neurocytol. 6: 195-210.

Cummings, J. P., and D. J. Stelzner (1984) Prenatal and postnatal development of lamina IX neurons in the rat thoracic spinal cord. Exp. Neurol. 83: 155-166.

Dennis, M. J., and A. J. Harris (1980) Transient inability of neonatal rat motorneurons to reinnervate muscle. Dev. Biol. 74: 173-183.

Friedlander, M. J., L. R. Stanford, and S. M. Sherman (1982) Effects of monocular deprivation on the structure-function relationship of individual neurons in the cat's lateral geniculate nucleus. J. Neurosci. 2: $321-330$.

Graybiel, A. M., and C. W. Ragsdale (1982) Pseudocholinesterase staining in the primary visual pathway of the macaque monkey. Nature 299: $439-442$.

Grouse, L. D., B. K. Schrier, and P. G. Nelson (1979) Effect of visual experience on gene expression during the development of stimulus specificity in cat brain. Exp. Neurol. 64: 354-364.

Hendry, S. H. C., and M. B. Kennedy (1986) Immunoreactivity for a calmodulin-dependent protein kinase is selectively increased in macaque striate cortex after monocular deprivation. Proc. Natl. Soc. Sci. USA 83: 1536-1540.

Hendry, S. H. C., S. Hockfield, E. G. Jones, and R. McKay (1984) Monoclonal antibody that identifies subsets of neurons in the central visual system of monkey and cat. Nature 307: 267-269.

Hockfield, S. (1987) A novel cerebellar neuron identified by a monoclonal antibody generated with immunosuppressive and rapid immunization strategies. Science 237: 67-70.

Hockfield, S., and R. D. G. McKay (1983) A surface antigen expressed by a subset of neurons in the vertebrate central nervous system. Proc. Natl. Acad. Sci. USA 80: 5758-5761.

Hockfield, S., and R. D. McKay (1985) Identification of major cell classes in the developing mammalian nervous sytem. J. Neurosci. 5: 3310-3328.

Hockfield, S., R. D. McKay, S. H. C. Hendry, and E. G. Jones (1983) A surface antigen that identifies ocular dominance columns in the visual cortex and laminar features of the lateral geniculate nucleus. Cold Spring Harbor Symp. Quant. Biol. 48: 877-889.

Hockfield, S., M. Sur, D. O. Frost, and R. McKay (1985) The expression of a $Y$-cell antigen is developmentally regulated in cat lateral geniculate nucleus. Invest. Ophthalmol. Vis. Sci. (Suppl.) 26: 287.

Hubel, D. H., and T. N. Wiesel (1970) The period of susceptibility to the physiological effects of unilateral eye closure in kittens. J. Physiol. (Lond.) 206: 419-436.

Hubel, D., and T. Wiesel (1985) Binocular interaction in striate cortex of kittens reared with artificial squint. J. Neurophysiol. 28: 10411059 .

MacAvoy, M. G., S. Hockfield, and M. Sur (1985) Development of antigen expression in a possible $\mathrm{Y}$-cell pathway through the cat lateral geniculate nucleus and visual cortex. Soc. Neurosci. Abstr. 11: 224.

McArdle, J. J. (1975) Complex end-plate potentials at the regenerating neuromuscular junction of the rat. Exp. Neurol. 49: 629-638.

McKay, R. D., and S. J. Hockfield (1982) Monoclonal antibodies distinguish antigenically discrete neuronal types in the vertebrate central nervous system. Proc. Natl. Acad. Sci. USA 79: 6747-6751.

Meyer, R. L. (1983) Tetrodotoxin blocks the formation of ocular dominance columns in goldfish. Science 218: 589-591.

Mower, G. D., W. F. White, and F. H. Duffy (1985) GABA receptor binding in normal and monocularly deprived cats. Invest. Opthalmol. Vis. Sci. (Suppl.) 26: 9. 
Phelps, P. E., R. P. Barber, C. R. Houser, G. D. Crawford, P. M Salvaterra, and J. E. Vaughn (1984) Postnatal development of neurons containing choline acetyltransferase in rat spinal cord: An immunocytochemical study. J. Comp. Neurol. 229: 347-361.

Ramon y Cajal, S. (1928) Degeneration and Regeneration of the Nervous System, Hafner, New York.

Ronnevi, L-O., and S. Conradi (1974) Ultrastructural evidence for spontaneous elimination of synaptic terminals on spinal motoneurons in the kitten. Brain Res. 80: 335-339.

Schmalbruch, H. (1984) Motorneuron death after sciatic nerve section in newborn rats. J. Comp. Neurol. 224: 252-258.

Sheard, P., McCaig, C. D., and A. J. Harris (1984) Critical periods in rat motor neuron development. Dev. Biol. 102: 21-31.

Sherman, S. M., K-P. Hoffman, and J. Stone (1972) Loss of a specific cell type from dorsal lateral geniculate nucleus in visually deprived cals. J. Neurophysiol. 35: 532-541.

Smith, D. E. (1974) The effect of deafferentation on the postnatal development of Clarke's nucleus in the kitten-a Golgi study. Brain Res. 74: 119-130.
Spemann, H. (1962) Embryonic Development and Induction, Hafner, New York.

Stryker, M. P., and W. A. Harris (1986) Binocular impulse blockade prevents the formation of ocular dominance columns in cat visual cortex. J. Neurosci. 6: 2117-2133.

Sur, M., D. O. Frost, and S. Hockfield (1988) Expression of a surfaceassociated antigen on $Y$-cells in the cat lateral geniculate nucleus is regulated by visual experience. J. Neurosci. 8: 874-882.

Wiesel, T. N. (1982) Postnatal development of the visual cortex and the influence of environment. Nature 299: 583-591.

Wiesel, T. N., and D. H. Hubel (1963) Single-cell responses in striate cortex of kittens deprived of vision in one eye. J. Neurophysiol. 26: 1003-1017.

Wong-Riley, M. T., and E. Carroll (1984) Effect of impulse blockage on cytochrome oxidase activity in monkey visual system. Nature 307 : 262-264.

Zaremba, S., and S. Hockfield (1987) Monoclonal antibody Cat-301 recognizes a proteoglycan specific to the surface of subsets of mammalian CNS neurons. Soc. Neurosci. Abstr. 13: 1228 